\title{
THERAPEUTIC OPTIONS USED FOR MYASTHENIA GRAVIS
}

Clinical and economic outcomes following plasma exchange (PLEX) and intravenous immunoglobulin (IVIG) in 1,606 hospitalized adult patients with myasthenia gravis (MG) were compared in a study at Case Western Reserve University, Cleveland, $\mathrm{OH}$; St Louis University, MO; and University of Alabama, Birmingham, AL. The cohort was identified from the Nationwide Inpatient Sample database for 2000-2005. Mean age was approx 50 years. MG crisis patients $(n=698)$ had higher mortality and complication rates, and those receiving PLEX $(n=529)$ had significantly more complications than those receiving IVIG (169). Mortality and complication rates of the treatment groups did not differ significantly in the whole cohort. Respiratory, cardiac, or renal failure was associated with increased mortality, and age and respiratory failure with increased complication rate. Patients receiving PLEX had longer length of hospital stay and higher inpatient costs. Conipared to PLEX, patients treated with IVIG have similar clinical outcome and lower costs of hospitalization. Elderly and those with acute respiratory or cardiac failure may respond better to IVIG. (Mandawat A, Kaminski HJ, Cutter G, Katirji B, Alshekhlee A. Comparative analysis of therapeutic options use for myasthenia gravis. Ann Neurol Dec 2010;68:797-805). (Respond: Dr Alshkhlee, Department of Neurology and Psychiatry, St Louis University, 1438 S Grand Blvd, St Louis, MO 63104).

COMMENT. Miller RG, Barohn RJ, and Dubinsky R, in an editorial, agree that IVIG and PLEX appear to be equivalent in efficacy, and IVIG may be preferable in tolerability and hospital costs. (Ann Neurol 2010;68:776-777). They outline the major therapeutic advances: physostignine and thymectomy (1930s), mechanical ventilation (1950s), corticosteroids and PLEX (1960s), azathioprine (1960s to 1970s), cyclosporine (1980s), IVIG (1980s to 1990s), and mycophenolate mofetil (1990s to 2000s). They note similarities between treatment of $\mathrm{MG}$ and Guillain Barre syndrome and chronic inflammatory demyelinating polyradiculoneuropathy, with respect to the efficacy of IVIG and PLEX. The response to thymectomy is not discussed, except that in adults it is disappointing and under investigation by controlled trial.

Tracy MM and colleagues at Children's Memorial Hospital, Chicago, reported a "Graded response to thymectomy in 13 children with MG" (J Child Neurol 2009;24:454-459). Thymectomy was considered an effective treatment in $62 \%$ of this series, and remission was complete with no medication in $31 \%$. A review of the literature from 1960 to 2009 uncovered 18 articles concerning response to thymectomy in children with MG, and a total of 479 patients of whom $68 \%$ showed improvement and $39 \%$ had discontinued medication.

\section{INFECTION-RELATED DISORDERS}

\section{KLUVER-BUCY SYNDROME AFTER MENINGOENCEPHALITIS}

Researchers at Medical College of Georgia, Augusta, GA, report a 10-year-old girl with a history of Listeria monocytogenes meningoencephalitis, contracted at $2 \frac{1}{2}$ years of age, who developed behavioral changes consistent with Kluver-Bucy syndrome 
at 2 months following discharge. Following a week of fever, vomiting, and diarrhea with dehydration, she developed status epilepticus. A postinfectious communicating hydrocephalus was treated with ventriculoperitoneal shunt. Two months after discharge, she gradually exhibited a behavioral change characterized by aggression toward family members and pets, uncontrolled screaming and crying, inability to sustain attention span, hyperactivity, and decreased language output. Persistent behavior problems were treated with clonidine and Concerta, and were complicated at 4 and $1 / 2$ years of age by hypersexuality (frequent masturbation), hyperorality, and significant weight gain. MRI at age 4 showed diffuse brain atrophy with maximal temporal lobe involvement and at 9 years, decreased hippocampal volume bilaterally. Complex partial seizures at 6 years of age were partially controlled with carbamazepine and lamotrigine. Except for tactile exploratory behavior and clinging to the examiner, the neurological exam was normal. EEG showed focal slowing bilaterally with epileptiform discharges over right and left midtemporal regions. Serial neuropsychological evaluations at 5,7 , and 10 years of age demonstrated expressive and receptive language impairment and an amnestic syndrome. She had poor working memory and verbal learning performance and was unable to make new declarative memories. Her reading, spelling, and arithmetic performance decreased from low average/average to borderline at age 10 years. Oral reading fluency, comprehension, and written expression were in deficient range. (Cohen MJ, Park YD, Kim H, Pillai JJ. Long-term neuropsychological follow-up of a child with Kluver-Bucy syndrome. Epilepsy Behav Dec 2010;19:643-646). (Respond: Dr Morris J Cohen, Medical College of Georgia, $112015^{\text {th }}$ Street, Bt-2601, Augusta, GA 30912).

COMMENT. Kluver and Bucy at the University of Illinois, Chicago, in 1937 and 1938, described a "psychic blindness" and other symptoms following bilateral temporal lobectomy in rhesus monkeys. Terzian et al reported the first case of KB syndrome in man in 1955, following bilateral removal of temporal lobes for intractable epilepsy. In adults, the characteristic symptoms of $\mathrm{KB}$ syndrome include 1) visual agnosia, inability to recognize objects without loss of visual discrimination; 2) hyperorality, urge to examine objects by mouth; 3) hypermetamorphosis, excessive attentiveness/touching of visual stimuli; 4) placidity, loss of normal fear or anger; 5) hypersexuality; and 6) changes in dietary habits. Also, aphasia, memory loss, and seizures occur in human cases. The syndrome is rare, only 27 pediatric cases reported, and is usually incomplete. Hyperorality and hypersexuality are most frequent features, followed by bulimia. Causes in children are various, including herpes simplex encephalitis, HIE, traumatic brain injury, neurocysticercosis, mycoplasma infection, bilateral arachnoid cysts, and encephalopathy.

\section{PREDICTORS OF MENINGITIS WITH FIRST FEBRILE SEIZURE}

Researchers in Delhi, India, investigated the prevalence of bacterial meningitis in 497 children aged 6-18 months admitted to hospital with a first seizure with fever. Lumbar puncture was performed in 199 (40\%) infants; 116 with "simple" seizures and 83 "complex." (The hospital has a policy of LP in all patients with complex febrile seizures). Meningitis was diagnosed in 5 infants $(2.4 \%) ; 1(0.86 \%)$ with simple, and $4(4.81 \%)$ with complex features. Risk factors for meningitis included 1) duration of seizure $>30 \mathrm{~min} ; 2$ ) 